\title{
Vom Röntgen zum PET/MRT, und dann? - Zukunftsweisende Bildgebung in der Kinderradiologie
}

\section{From Xrays to PET/MR, and then? - Future imaging in pediatric radiology}

Autoren

Gundula Staatz ${ }^{1}$, Heike Elisabeth Daldrup-Link ${ }^{2}$, Jochen Herrmann ${ }^{3}$, Franz Wolfgang Hirsch ${ }^{4}$, Jürgen F. Schäfer ${ }^{5}$, Anna Seehofnerova ${ }^{6}$, Erich Sorantin ${ }^{6}$, Ashok Joseph Theruvath ${ }^{2,7}$, André Lollert ${ }^{1}$

Institute

1 Department of Diagnostic and Interventional Radiology, Section of Pediatric Radiology, Medical Center of the Johannes-Gutenberg-University Mainz, Germany

2 Department of Radiology, Stanford-University Medical Center, Stanford, California, USA

3 Section of Pediatric Radiology, Department of Diagnostic and Interventional Radiology and Nuclear Medicine, University-Medical-Center Hamburg-Eppendorf, Hamburg, Germany

4 Department of Pediatric Radiology, University-Hospital Leipzig, Germany

5 Department of Diagnostic and Interventional Radiology, Section of Pediatric Radiology, University-Hospital Tübingen, Tübingen, Germany

6 Division of Pediatric Radiology, Department of Radiology, Medical University Graz, Austria

7 Department of Diagnostic and Interventional Radiology, Medical Center of the Johannes-Gutenberg-University Mainz, Germany

Bibliografie

DOI https://doi.org/10.1055/a-0853-2744

Online-Publikation: 2019

Fortschr Röntgenstr 2019; 191: 357-366

(c) Georg Thieme Verlag KG, Stuttgart · New York ISSN 1438-9029

Korrespondenzadresse

Univ.-Prof. Dr. med. Gundula Staatz

Department of Diagnostic and Interventional Radiology

Section of Pediatric Radiology

Medical Center of the Johannes-Gutenberg-University,

Langenbeckstr. 1, 55131 Mainz, Germany

Tel.: ++49/6131/176927

Fax: ++49/6131/176682

gundula.staatz@unimedizin-mainz.de

\section{ZUSAMMENFASSUNG}

Die Kinderradiologie steht heute vor weitreichenden Veränderungen. Basismodalitäten wie Röntgen und Ultraschall werden zunehmend durch neuere, moderne Techniken ergänzt. Dieser Übersichtsartikel stellt Fortschritte in der Kinderradiologie sowie technische Innovationen vor, welche in Zukunft noch größere Bedeutung erlangen könnten. Hierzu werden CT-Dosisreduktionstechniken inklusive der Anwendung künstlicher Intelligenz sowie Fortschritte in den Gebieten der Magnetresonanztomografie und molekularen Bildgebung dargestellt.

\section{Kernaussagen}

- Technische Innovationen werden die Kinderradiologie signifikant verändern.

- CT-Dosisreduktion ist insbesondere in pädiatrischen Patientenkollektiven bedeutsam.

- Neue MR-Techniken werden die Notwendigkeit von Sedierung und Kontrastmittelapplikation reduzieren.

- Die funktionelle MR-Bildgebung kann bei Patienten mit chronischen Lungenerkrankungen an Bedeutung gewinnen.

- Die molekulare Bildgebung ermöglicht die Detektion, Charakterisierung und Quantifizierung molekularer Prozesse in Tumoren.

\section{ABSTRACT}

Significant changes can be expected in modern pediatric radiology. New imaging techniques are progressively added to basic modalities like Xrays and ultrasound. This essay summarizes recent advances and technical innovations in pediatric radiology, which are supposed to gain further importance in the future. Thus, CT dose reduction techniques including artificial intelligence as well as advances in the fields of magnetic resonance and molecular imaging are presented.

\section{Key Points}

- Technical innovations will lead to significant changes in pediatric radiology.

- CT dose reduction is crucial for pediatric patient collectives.

- New MR-techniques will lower the need for sedation and contrast media application.

- Functional MR-imaging might gain further importance in patients with chronic lung disease.

- Molecular imaging enables detection, characterization and quantification of molecular processes in tumors.

\section{Citation Format}

- Staatz G, Daldrup-Link HE, Herrmann J et al. From Xrays to $\mathrm{PET} / \mathrm{MR}$, and then? - Future imaging in pediatric radiology. Fortschr Röntgenstr 2019; 191: 357-366 


\section{Einleitung}

Für die Kindermedizin bedeutete die Röntgentechnik, die erstmals 1897 in einer Kinderklinik installiert wurde, einen sensationellen Einschnitt: Die Tuberkulosediagnostik wurde auf eine objektivierbare Basis gestellt. Röntgenbefunde markierten damit den Beginn einer evidenzbasierten Kindermedizin. Die am häufigsten durchgeführte Bildgebung im Kindesalter wurde jedoch die Ultraschalldiagnostik, für die Kinderärzte und Kinderchirurgen frühzeitig den hohen Stellenwert erkannten.

Ein weiterer relevanter Entwicklungsschritt war die Einführung der Computertomografie (CT), welche erstmals die Notwendigkeit eines spezialisierten Kinderradiologen erforderlich machte.

Obwohl zeitgleich mit der CT-Diagnostik entwickelt, war eine breite Verfügbarkeit der Magnetresonanztomografie (MRT) für die Kinderradiologie erst ab Mitte der 1990er Jahre gegeben.

Mit der MRT stand der Kinderradiologie eine neue Modalität zur Verfügung, die gerade von jungen Radiologen als attraktiv bewertet wird und Forschungspotenzial hat. Mit dem Hybrid-Verfahren der Positronen-Emissions-Tomografie (PET)/MRT kam ab 2013 nun eine zusätzliche MRT-Variante hinzu, die auch von den Kinderradiologen mitgetragen wird. Bei den meisten Hybrid-Anwendungen wäre das PET/CT sowohl schneller als auch diagnostisch ausreichend. Das PET/MRT begründet dagegen seine klinische Anwendung aber mit einem relevanten Strahlenschutzaspekt.

\section{Low-dose-CT - Wie wenig Dosis wird möglich sein?}

Neue Detektor- und Bildrekonstruktionstechnologien sowie Computeranwendungen erlauben deutliche Dosissenkungen in der $\mathrm{CT}$. Aktuell sind Begriffe wie „Artificial Intelligence (AI)“ und dessen Subbegriff „Deep Learning (DL)“ zu hoffnungsfroh assoziierten Schlagworten geworden - bekannt sind u. a. Anwendungen von $\mathrm{Al}$ in der Bildsegmentierung/Klassifizierung [1, 2].

Im Bereich der Bildrekonstruktion sind erste Applikationen berichtet worden mit einer Verbesserung der „Peak Signal to Noise Ratio“ von 23 \% [3]. Hier wird die Zukunft zeigen, ob man mithilfe von Al und DL die Dosis senken oder die Scangeschwindigkeit durch Reduktion der Projektionen reduzieren kann.

Es scheint unstrittig, dass sich auch die Kinderradiologen zügig den Al-Techniken zuwenden müssen. Wir müssen uns auf eine grundlegende Veränderung auch unseres Befundungs-Verhaltens und damit auch des ganzen Berufsbildes einstellen. Der Radiologe wird dann letztlich entscheiden müssen, welche Struktur in der Untersuchung „real“ oder ein mathematisches Konstrukt (= Artefakt) ist. Geprüfte kinderradiologische Bilddatensätze müssen den Entwicklern von Kinderradiologen selbst zur Verfügung gestellt werden, um neue Anwendungen zu generieren.

Um auszuloten, wie weit man die Dosis bei der CT senken kann, veröffentlichten Messerli et al. ein „Ultra-Low-Dose-CT“ (Dosis gleich der eines Lungenröntgens bei Erwachsenen $=0,13 \mathrm{mSv}$ ) für die Detektion von Lungenrundherden bei Erwachsenen [4]. In der Radiologie Graz wurde anhand einer Phantomstudie (4 Jahre alter Knabe - http://www.kyotokagaku.com/products/detail03/ ph-2c.html) versucht, diese Ergebnisse am eigenen CT-Gerät
(AquilionONE, Canon Medical Systems Europe B.V., NL) nachzuvollziehen. Die Effektivdosis [5] eines Thorax-Röntgens in einer Ebene beträgt in der Abteilung 0,01 mSv und würde unter Berücksichtigung der altersentsprechenden Konversionsfaktoren [6] einem Dosis-Längenprodukt (DLP) von ca. 0,30 mGy · cm entsprechen. Das entsprechende DLP dieser Effektivdosis war beim vorhandenen CT-Gerät nicht einzustellen, sondern nur ein DLP von 3,8 $\mathrm{mGy} \cdot \mathrm{cm}$ - die Effektivdosis entspricht dann etwa 13 Thorax-Aufnahmen dieser Altersgruppe in einer Ebene. In $>$ Abb. 1 finden sich axiale Schichten des Phantoms von der geringsten Effektivdosis bis zu der in der Routine verwendeten. Es ist zu erkennen, dass in diesem Phantom auch bei der geringsten Dosis die Lungenstrukturen zu erkennen sind. Bei einem Hersteller, der Einstellungen der Röhrenspannung von 70 kV erlaubt, könnte dann bei allen gleichbleibenden Faktoren eine weitere Dosisreduktion von 25 \% im Vergleich zu 80 kV ermöglicht werden, entsprechend einer Effektivdosis von ca. 10 Thorax-Röntgen.

Es ist zu spekulieren, dass durch neue Detektormaterialien, Fortschritte in der statistischen Rekonstruktion, Einstellmöglichkeiten von 60 kV für Röhrenspannung und Röhrenstrom von weniger als $10 \mathrm{~mA}$ diagnostisch verwertbare CT-Untersuchungen mit einer vergleichbaren Effektivdosis von 3-5 Thorax-Aufnahmen anzufertigen sind.

Unter Beachtung einiger weniger Faktoren sind mit jedem CT Dosiseinsparungen in Höhe von 50 \% schon derzeit möglich mit immer noch „Luft nach oben“ $[5,7]$.

\section{MRT-Techniken der Zukunft in der pädiatrischen Radiologie}

Zukunftsweisende Konzepte zielen darauf ab, die Akzeptanz der MRT-Untersuchung zu verbessern und die Methodik für ein erweitertes Indikationsspektrum mit konkreten, klinisch relevanten Endpunkten zu etablieren [8]. Mithilfe einer Kombination aus pädagogisch-spielerischen Ansätzen und neuen MRT-Techniken lässt sich das Risikoprofil der MRT verbessern und hierdurch die Notwendigkeit einer Sedierung und die Verwendung von Gadolinium-haltigen Kontrastmitteln minimieren. Mittels neuer Sequenztechniken kann die Untersuchungsdauer verkürzt, eine erhöhte Robustheit gegenüber Bewegung erreicht und Kontrastmittel vermieden werden $[9,10]$.

\section{Beschleunigung}

Die Beantwortung komplexer Fragestellungen im Rahmen pädiatrischer MRT-Untersuchungen führt häufig zu langen Untersuchungszeiten. Mit der Dauer der MRT steigt die Wahrscheinlichkeit von Artefakten durch Bewegung deutlich an. MRT-Techniken wie die Multislice Akquisition [11 - 13] und das Compressed Sensing, einer Kombination aus paralleler Bildgebung mit einem rekonstruktionsbasierten iterativen Komprimierungsalgorithmus, können die Dauer einzelner Sequenzen und somit die Gesamtuntersuchungsdauer deutlich verkürzen (30 bis $50 \%$ ) [14]. 


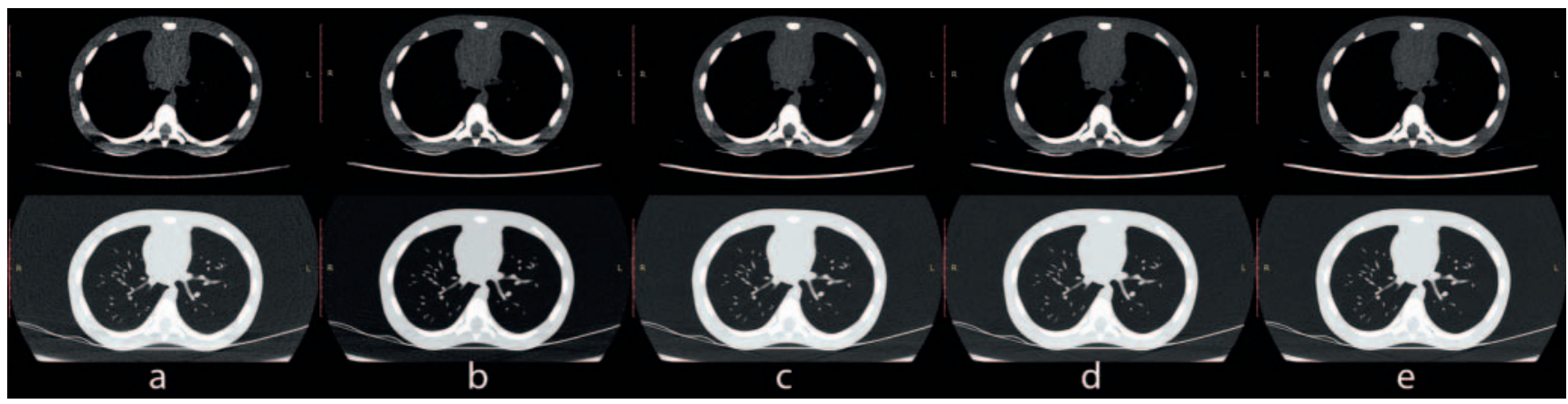

- Abb. 1 CT eines Thorax-Phantoms einem Kind im Alter von 4 Jahren entsprechend - die obere Zeile enthält axiale Schichten rekonstruiert im Weichteil-Kernel, die untere Reihe die gleiche Schichtposition im Lungen (= kantenbetonten) -Kernel. Die Effektivdosis steigt von A-E, und ist in A die geringste einstellbare - A 0,13 mSv, B 0,26 mSv, C 0,39 mSv, D 0,52 mSv und E 0,59 mSv.

\section{Bewegungsstabilität}

Bewegung während der Akquisitionsphase führt zu Artefakten und schränkt die Qualität pädiatrischer MRT-Untersuchungen häufig ein. Durch nichtkartesisches K-Raum-Sampling (radial, spiral) können bewegungsstabilere Ergebnisse erzielt werden [9, 15]. Die Technik kann in der 2D- und als 3D-Sequenz für die Akquisition von Volumendatensätzen angewendet werden und steht u. a. für T1- und T2-Kontraste zur Verfügung. Das Verfahren eignet sich auch für dynamische T1-gewichtete Untersuchungen des Abdomens nach Kontrastmittelgabe [16].

\section{Kontrastmittelfreie Untersuchung}

Zur Charakterisierung von entzündlichen und tumorassoziierten Veränderungen, Darstellung von Gefäßen und der Gewebeperfusion werden intravenöse, Gadolinium-haltige Kontrastmittel eingesetzt. Aufgrund des Risikoprofils soll die Kontrastmittelgabe in der Pädiatrie auf ein Minimum beschränkt werden. Zu den neuen alternativen, kontrastmittelfreien MR-Techniken zur Gewebecharakterisierung zählt die molekulare APT-Bildgebung (Amide-Proton-Transfer) [17, 18]. Kontrastmittelfreie 3D-Angiografien sind in hochauflösender Technik und freier Atmung beispielsweise mittels REACT-Technik (Relaxation-enhanced MR-Angiografie) zu realisieren [19]. Eine Analyse von komplexen intravaskulären Strömungssituationen kann über eine 4D-Flussdarstellung erfolgen und mithilfe neuer ultraschallbasierter Trigger-Verfahren bereits pränatal eingesetzt werden ( $\triangleright$ Abb.2) [20-22].

\section{Quantitative Techniken}

In Ergänzung zur konventionellen MRT finden zunehmend auch quantitative Techniken zur genaueren Gewebecharakterisierung Einzug in Forschung und Klinik, exemplarisch die Messung von T1- und T2-Relaxationszeiten, Diffusions-Bildgebung, Eisen-/Fettquantifizierung, Elastografie etc. [23-25]. Die in Entwicklung befindliche „MR-Fingerprinting“-Technik könnte in Zukunft die quantitative MRT deutlich beschleunigen, indem multiple Parameter simultan in einer Messung erfasst werden [26, 27]. Mittels Pseudo-Randomisierung von Sequenzparametern wie Repetiti- onszeit und Flip-Winkel und nachgeschaltetem Matching der Sequenzrohdaten mit einer Datenbank („Voxel-Fingerprint“) ist eine Gewebeerkennung und -charakterisierung möglich [28].

\section{Funktionelle MRT der Lunge bei Kindern}

In Konkurrenz zur CT oder Szintigrafie ist die MRT der Lunge bei Kindern aufgrund der fehlenden Strahlenexposition vielversprechend. Durch Innovationen im Bereich der Sequenzmethoden und der Nachverarbeitung wurden die Bildqualität und Reproduzierbarkeit der Ergebnisse verbessert. Insbesondere Veränderungen der kleinen Atemwege können nur mit funktionellen MR-Methoden untersucht werden [29]. Gerade die Beurteilung der regionalen Funktion ist aufgrund der höheren Sensitivität im Vergleich zu globalen Lungenfunktionstests für das Monitoring und Interventionen - z. B. bei zystischer Fibrose (CF) - von großer klinischer Relevanz [30]. Verschiedene Ansätze wurden bei Kindern untersucht, um Ventilation und Perfusion mit der MRT abzubilden.

\section{Inhalation von Gasen}

Durch Polarisation der Edelgase ${ }^{3} \mathrm{HE}$ und dem besser verfügbaren ${ }^{129} \mathrm{XE}$ lässt sich ein ausreichendes MR-Signal erzeugen, sodass neben der statischen Verteilung dynamische Messungen und u.a. der regionale Sauerstoffpartialdruck erfasst werden können [31]. Dem Vorteil der guten Löslichkeit in Blut von ${ }^{129} \mathrm{XE}$ und der damit verbundenen Möglichkeit, den Gasaustausch zu quantifizieren, steht die anästhetische Wirkung gegenüber. Untersuchungen bei gesunden Kindern und Patienten mit CF zeigen aber, dass dieser Effekt nur gering und von kurzer Dauer ist [32]. Nicht zuletzt der hohe technische Aufwand sowie die Kosten bei ${ }^{3} \mathrm{HE}$ stehen jedoch einer weiten klinischen Verbreitung der Methoden entgegen.

Eine kostengünstige, bisher nur bei Erwachsenen untersuchte Alternative besteht in Form der fluorierten Gase [33]. Sowohl Vorkommen als auch fehlende Toxizität von Fluor sprechen für den klinischen Einsatz, allerdings müssen ${ }^{19} \mathrm{~F}$-taugliche Spulen bzw. Multikernspulen genutzt werden.

Bei Inhalation des molekularen Sauerstoffs als paramagnetisches Kontrastmittel (O2-MRT) hängt die regionale Sig- 

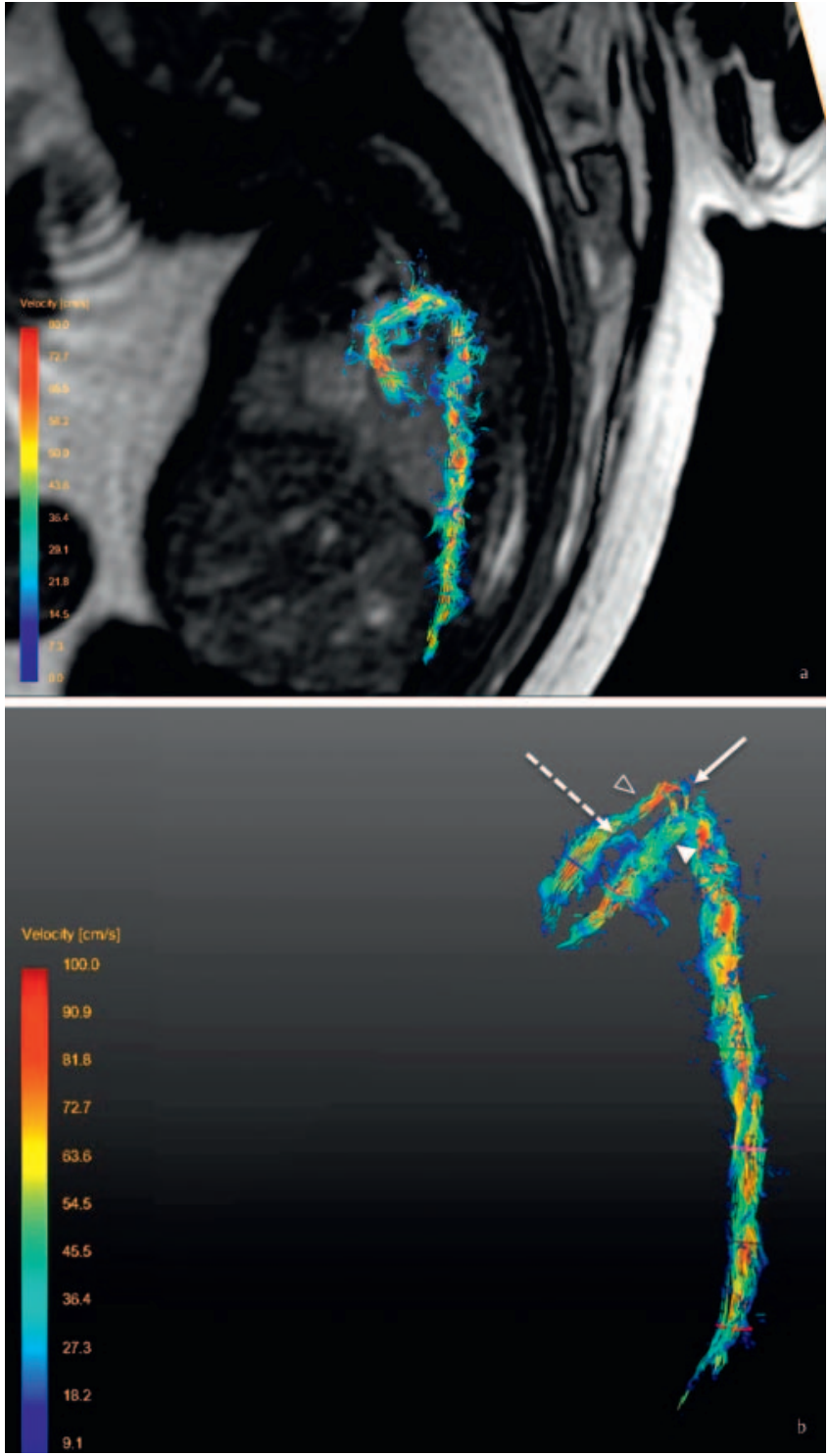

- Abb. 2 4D-Fluss-MRT-Bildgebung bei einem Fetus der 34 Schwangerschaftswoche in parasagittaler Schnittbildführung als Überlagerung einer T2-HASTE $\mathbf{A}$ und als Einzelbild $\mathbf{B}$. Dargestellt ist der Ductus arteriosus (Pfeil) zwischen A. pulmonalis (Pfeilspitze) und Aorta ascendens (Pfeilspitze ohne Füllung). Zusätzlich sichtbar ist das Foramen ovale (gestrichelter Pfeil). Abbildung zur Verfügung gestellt von PD Dr. med. Schönnagel, UKE, Hamburg.

nalintensität sowohl von Ventilation, Sauerstoffdiffusion und Lungenperfusion ab. Trotz guter Daten für die regionale Funktionsanalyse bei gleichzeitiger methodischer Verbesserung und minimaler Kosten konnte sich die O2-MRT klinisch bisher nicht durchsetzen [34]. Neben langen Messzeiten sind der Methode durch Maskeninhalation von reinem Sauerstoff im Wechsel mit Raumluft in der Anwendung bei Kindern Grenzen gesetzt.

\section{Dynamische, kontrastmittelverstärkte MRT}

Die gut etablierte dynamische, kontrastmittelverstärkte MRT (dynamic contrast-enhanced MRI; DC-MRI) bietet nicht nur die visuelle Beurteilung der regionalen Perfusion, sondern es können mittels Indikatordilutionsmethode die mittlere Transitzeit, das pulmonale Blutvolumen und der Blutfluss kartiert werden [35]. Da Ventilationsdefekte zu einer hypoxisch bedingten lokalen Vasokonstriktion (v. Euler und Liljestrand) führen, wird so auch eine Beurteilung der Obstruktionen der kleinen Atemwege möglich, bevor morphologische Veränderungen mit der MRT erkennbar sind [36]. So lässt sich z. B. bei CF der positive Effekt einer Antibiotikatherapie sehr sensitiv mit der DC-MRI nachweisen [37].

\section{Nichtinvasive funktionelle MR-Methoden}

Da nicht zuletzt der Einsatz Gadolinium-haltiger Kontrastmittel bei Kindern besonderen Indikationen vorbehalten sein sollte, konzentrieren sich die Entwicklungen auch auf nichtinvasive funktionelle MR-Methoden. Hierzu zählt das arterial spin labeling (ASL) mit magnetischer Markierung des frei diffundierenden Wassers des fließenden Blutes [38] ( $\bullet$ Abb. 3). Trotz Anfälligkeit für Artefakte können auch im Kindes- und Jugendalter repräsentative 2D-Perfusionsmaps erstellt werden [39]. Da ein Zusammenhang zwischen pulmonalem Volumen und MRT-Signal besteht, kommen weitere Methoden infrage.

Die Fourier-Dekompositions-MRT (FD-MRT) basiert auf der nativen Messung der gleichzeitig stattfindenden periodischen Signaländerung durch Pulsation der Lungenarterien und der Respiration. Mittels einer Registrierung auf ein Referenzbild und anschließender Fourier-Analyse lassen sich damit sowohl Ventilations- als auch Perfusionsmaps erstellen [40]. Das anfänglich zeitaufwendige 2D-Verfahren ließ sich durch ultraschnelle SSFPSequenzen verbessern, wodurch sich neben visuellen auch quantitative Ansätze ergeben [41].

Auch eine direkte Visualisierung des Protonengehalts ist sehr erfolgversprechend. Hierzu ist unter Hochfeldbedingungen eine Messung mittels sehr kurzer oder ultrakurzer Echo-Zeit (very short, ultra short TE; UTE) notwendig [42]. Mittlerweile stehen 3D-Verfahren zur Verfügung, die sowohl statische als auch Messungen unter Atmung erlauben. Vielversprechende erste Ergebnisse liefern eine gute Übereinstimmung sowohl visueller als auch funktioneller Parameter mit CT und Lungenfunktionstests ( $\bullet$ Abb. 4) $[43,44]$. Wenn sich Robustheit und Sensitivität der Methode bestätigen, wird dies einer breiten Anwendung in der Kinderradiologie zugutekommen.

\section{Pädiatrische molekulare Bildgebung}

Die molekulare Bildgebung kann bestimmte Zielzellen oder Zielmoleküle in Tumoren erkennen, indem sie Gen- und Proteinfunktionen und die daraus resultierenden Veränderungen des Gewebestoffwechsels in vivo markiert [45]. Damit kann die molekulare Bildgebung spezifischere Informationen über Tumoren liefern als herkömmliche bildgebende Verfahren, die auf passiver Gewebeanreicherung von Kontrastmitteln beruhen [45]. Biomarker der molekularen Bildgebung können pathologische Prozesse quantifizieren, die zur Früherkennung von Tumoren, zur Einschätzung der Tumor-Aggressivität und zur Überwachung der Therapie-Respon- 


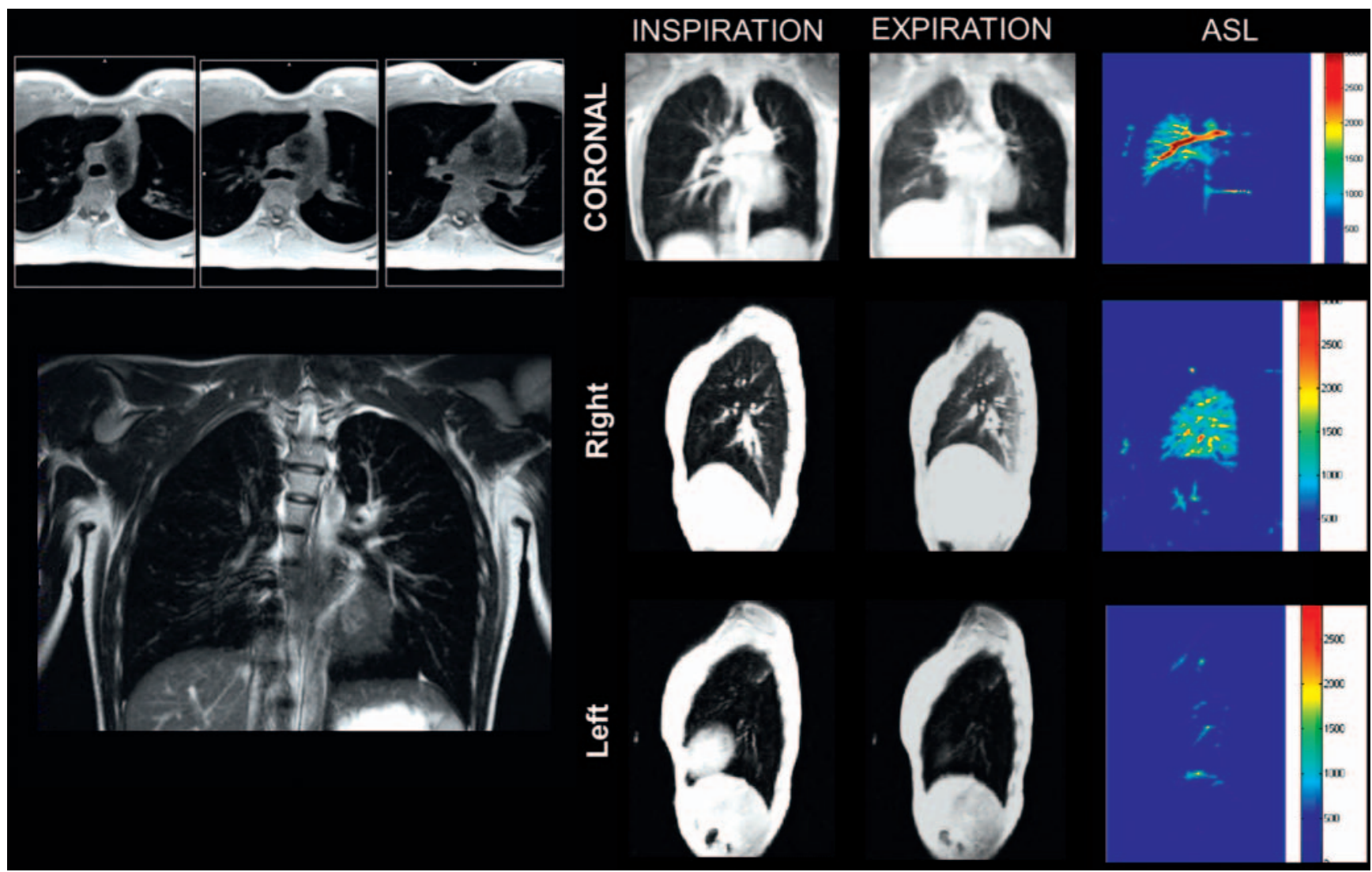

Abb. 3 Lungen-MRT bei Swyer-James-Syndrom, 16-jährige Patientin, anhaltende Dyspnoe bei Zustand nach Pneumonie. Links: Morphologische Bildgebung (VIBE-Sequenzen oben, T2w-TSE unten) ohne Hinweis auf eine Obstruktion der großen Bronchien. Mitte: Die dynamische Untersuchung in In- und Exspiration (2D-Gradientenecho mit sehr kurzer Echo-Zeit) zeigt eine deutliche Überblähung der gesamten linken Lunge. Rechts: Arterial-spin labeling (flow-sensitive alternating inversion recovery HASTE). Nachweis der hochgradigen Minderperfusion der linken Lunge.

se genutzt werden können [46-48]. Eine Vielzahl von molekularen Bildgebungs-Verfahren werden bereits in der Klinik eingesetzt, während andere noch in der Testphase sind [48].

\section{Ultraschall}

Neue Ultraschall-Kontrastmittel (microbubbles) werden inzwischen von vielen Zentren verwendet und haben die Spezifität von Ultraschalluntersuchungen für die Diagnose von Tumoren und Entzündungen verbessert $[49,50]$. Allerdings sind herkömmliche Ultraschall-Kontrastmittel nicht spezifisch. Da die meisten $\mu \mathrm{m}$ großen Mikroblasen (microbubbles) im intravaskulären Raum verbleiben und nicht in das Interstitium von Tumoren gelangen, können Mikroblasen vornehmlich Rezeptoren auf der intraluminalen Gefäßoberfläche markieren [51]. Willmann und Mitarbeiter haben gezeigt, dass Mikroblasen den kinase insert domain receptor (KDR) in Mamma- und Ovarial-Karzinomen von erwachsenen Patienten markieren und mit Ultraschall in vivo nachweisen konnten [46]. Diese Studie zeigt das Potenzial der Ultraschall-Bildgebung für rezeptor-spezifische Tumor-Bildgebung auch im Hinblick auf pädiatrische Patienten.

\section{Optische Bildgebung}

Die optische Bildgebung (optical imaging, Ol) basiert auf der Erkennung von sichtbarem Licht (Fluoreszenz, Biolumineszenz, Absorption oder Reflexion). OI ist aufgrund seiner schnellen (<1 min) und strahlenfreien Bilddatenerfassung sehr attraktiv für pädiatrische Bildgebungsanwendungen. Darüber hinaus ist OI preiswert, nichtinvasiv und verfügt über molekulare Sensitivität [45, 52]. Zu den Einschränkungen der Ol gehören eine begrenzte Durchdringungstiefe, die begrenzte anatomische Auflösung und komplexe Algorithmen zur Datenquantifizierung [45]. Mindestens ein fluoreszierendes Kontrastmittel, indocyanine green (ICG), ist zugelassen für die medizinische Bildgebung [53]. Beispiele für klinische Ol-Anwendungen sind die Diagnose von Retina-Erkrankungen [54], intraoperative Erkennung von Peritoneal-Metastasen und die intraoperative Gehirntumorabgrenzung in Kombination mit neuronaler Bildgebung [52].

Es gibt auch Ansätze für Hybrid-Bildgebung mittels OI und konventioneller Radiografie, Ultraschall, MRT und SPECT/PET [45]. Für diese Hybrid-Technologien wurden multifunktionale Kontrastmittel entwickelt, die eine Validierung der Biomarker-Verteilung oder -aktivierung ermöglichen. So könnten beispielsweise PETDaten verwendet werden, um die Konzentration eines tumorspezifischen Biomarkers zu verfolgen und zu quantifizieren, während 


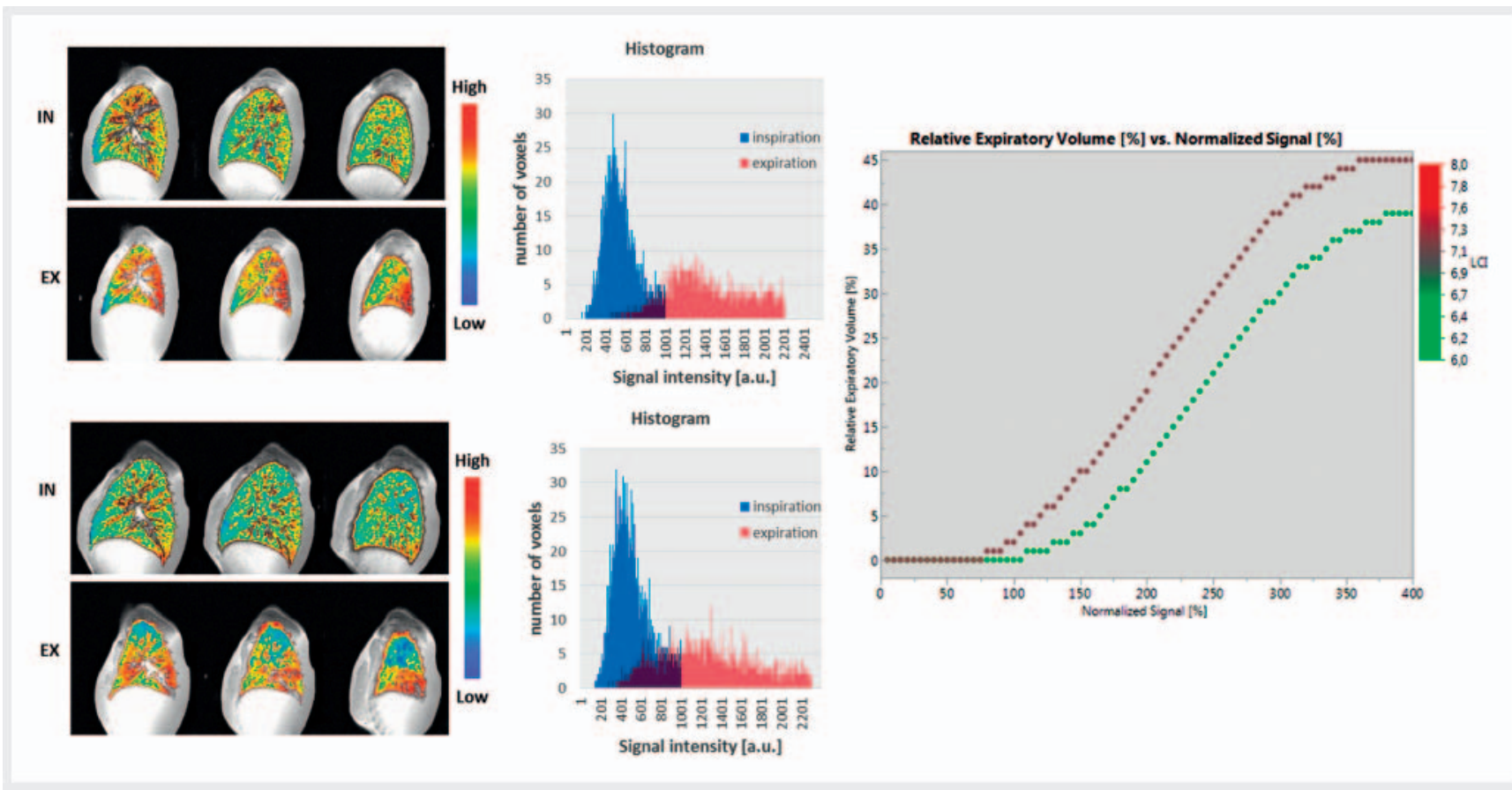

- Abb.4 Ventilationsmessung mittels sehr kurzer Echo-Zeit bei CF. Oben: 13-jähriger Patient mit normalem Lung-clearance-index (LCI). Unten: 16-jährige Patientin mit grenzwertig erhöhtem LCI. Links: Repräsentative, nach Signal-Intensität farbkodierte Schichten in In- und Exspiration der rechten Lunge von beiden Patienten. Bei normalem Muster oben findet sich unten eine verminderte Signalintensität (blau) des Oberlappens bei Überblähung. Mitte: Die jeweiligen Histogramme in In- und Exspiration. Rechts: Kumulative Histogramme des prozentualen exspiratorischen Volumens nach Normalisierung der Signalintensität (SI). Beide Darstellungen zeigen Unterschiede mit erhöhtem Volumen bei niedriger SI der 16-jährigen CF-Patientin (rote Kurve).

begleitende Fluoreszenzmarker eine enzymatische Aktivierung darstellen können.

\section{Fotoakustische Bildgebung}

Die fotoakustische Bildgebung ist eine neue Technologie, die auf der Aussendung von Laserlicht, das im Gewebe absorbiert und in Wärme umgewandelt wird, basiert. Dies führt zu einer thermoelastischen Ausdehnung des Zielgewebes und zur Emission von Ultraschallwellen, die wiederum mit einem Ultraschallgerät nachgewiesen werden können („Licht rein - Ultraschall raus"). Eine potenziell wichtige Anwendung für pädiatrische Patienten ist die fotoakustische Messung von Gewebe-Hämoglobin-Konzentrationen und Sauerstoffsättigungen. Diese Anwendung könnte z. B. für die nichtinvasive Überwachung der Oxygenierung des Gehirns und der Viszeral-Organe bei Frühgeborenen eingesetzt werden [8].

\section{Positronen-Emissionstomografie (PET) und PET/CT}

PET und PET/CT sind seit vielen Jahren für die molekulare Bildgebung von pädiatrischen Tumoren etabliert [55]. Zu den Vorteilen gehören die hohe Empfindlichkeit (Erkennung von PikomolarKonzentrationen) und ein hoher Tumor-zu-Hintergrund-Kontrast. 18F-2-Fluoro-2-Deoxy-Glukose (FDG) ist der klinisch etablierte
Tracer für die Tumor-Bildgebung bei Kindern mit Lymphomen, Knochensarkomen, Rhabdomyo-Sarkomen und Karzinomen [55]. Mehrere Studien zeigten eine höhere Sensitivität der 18F-FDGPET/CT im Vergleich zu allen kollektiven Standarduntersuchungen [56-60].

18F-FDG wurde auch als potenzieller Biomarker für das Ansprechen von Ewing-Sarkomen auf neue Antikörper untersucht, die insulinähnliche Wachstumsfaktor-Rezeptoren (IGF-R) blocken. IGF-1 und IGF-2 sind Polypeptide, die die Zellvermehrung und -differenzierung regulieren. Die Überexpression von IGF-1 R und IGF-2 R wurde bei Knochensarkomen (IGF-1 R) und RhabdomyoSarkomen (IGF-2 R) festgestellt. In präklinischen Studien verhinderten Antikörper gegen IGF-1 R/Insulin-Rezeptoren die Aufnahme von 18F-FDG in Tumorzellen. Eine klinische Studie an Erwachsenen zeigte, dass die FDG-PET mit hoher Sensitivität ein Ansprechen von Sarkomen auf Antikörper gegen IGF-R innerhalb 1 Woche nach Start der Therapie darstellen konnte. Neuere, klinisch relevante Tracer sind 18F-deoxy-fluorothymidine (FLT), ein Thymidin-Analog, für die Diagnose von Hirntumoren, sowie 124I-MIBG PET und 18F-DOPA PET/CT für die Detektion von Neuroblastomen [61, 62].

Eine Limitation der PET-Bildgebung ist die Unfähigkeit zur simultanen Erkennung von mehr als einem Tracer. Die relative hohe Strahlenexposition der PET/CT ist für die pädiatrische Bildgebung relevant, sodass die PET/MRT eine interessante Alternative darstellt. 


\section{MRT und PET/MRT}

Die hyperpolarisierte MRT und spektroskopische Bildgebung ist ein wichtiger, relativ neuer Bereich der molekularen MR-Bildgebung, der derzeit in präklinischen Studien und an erwachsenen Patienten erforscht wird [63-66]. Für klinische Anwendungen werden 13C-markierte Moleküle mit einem „dynamischen nuklearen Polarisierungssystem" (DNP) zu einer antiparallelen Orientierung polarisiert. Die Hyperpolarisierung des Moleküls zerfällt innerhalb von wenigen Minuten. Daher ist der Transport zum Patienten, die intravenöse Injektion und Bildgebung extrem zeitsensitiv. Jede zeitliche Verzögerung verhindert die Erfassung hyperpolarisierter MRT-Daten, was eine große Herausforderung für pädiatrische Anwendungen darstellt. Dennoch ermöglicht die hyperpolarisierte MRT, wenn erfolgreich, eine dynamische Kartierung von wichtigen biologischen Prozessen. Zum Beispiel kann die 1 - 13C-Pyruvat-hyperpolarisierte MRT Stoffwechselprodukte des Warburg-Zyklus messen. Verschiedene neue Chemotherapeutika, wie die Retiniod-Säure, führen zu einer Differenzierung von Tumorzellen zu Zellen, die sich nicht weiter duplizieren (anstatt einer Apoptose oder Nekrose). Die Retiniod-Säure invertiert den typischen Tumor-Stoffwechsel (erhöhte aerobe Glykolyse und verminderte oxidative Phosphorylierung = Warburg-Effekt), der mit der hyperpolarisierten MRT gemessen werden kann. Ein großer Vorteil der 13C-hyperpolarisierten Moleküle ist, dass sie keine bekannten biologischen Nebenwirkungen haben.

Die integrierte PET/MRT kombiniert die hohe anatomische Auflösung und den hohen Weichteilkontrast der MRT mit der hohen Empfindlichkeit der PET-Bildgebung und überwindet damit die eingeschränkte Empfindlichkeit der traditionellen MRT-Kontrastmittel und die relativ hohe Strahlenbelastung der PET/CT $[67,68]$. Sie ist daher in besonderem Maße für onkologische Verlaufskontrollen geeignet ( $\triangleright$ Abb. $\mathbf{5}$ ).

Eine weitere, direkt klinisch anwendbare molekulare MR-Bildgebungsmethode ist die Ganzkörper-MRT mit diffusionsgewichteter Bildgebung [69, 70]. Die diffusionsgewichtete GanzkörperMRT kann einen ähnlichen Tumor-zu-Hintergrundkontrast und einen besseren Weichteilkontrast als die 18F-FDG-PET-Scans bieten [70]. Diese strahlungsfreie Methode hat sich insbesondere für das Ganzkörperscreening von Patienten mit Tumor-Prädispositionssyndromen (z. B. p53-Mutationen, Li-Fraumeni-Syndrom, Hemihypertrophie, Neurofibromatose [45]) durchgesetzt. In $>$ Abb. 6 ist ein Beispiel einer PET/MRT mit integrierter diffusionsgewichteter Bildgebung anhand eines Patienten mit M. Hodgkin dargestellt.

Mehrere Studien haben gezeigt, dass FDG-PET-Untersuchungen ein Ansprechen von kindlichen Lymphomen und Sarkomen auf Chemotherapie zeigen können, bevor die Tumoren eine Änderung des Volumens zeigen [68, 71 - 73]. Bei Patienten mit Osteosarkomen konnte ein Ansprechen der Chemotherapie zuverlässig anhand von FDG-PET-Scans vorhergesagt werden und korrelierte mit einer Tumornekrose in der Histologie [49].

Es scheint einen Zusammenhang zwischen Tumorzellzahl und Glukosestoffwechsel zu geben: Mehrere Autoren haben beschrieben, dass ein Tumoransprechen auf Chemotherapie zu zunehmenden ADC (apparent diffusion-coefficient) -Werten und abnehmender Tumor-18F-FDG-Aufnahme führt [74]. Die Messung der

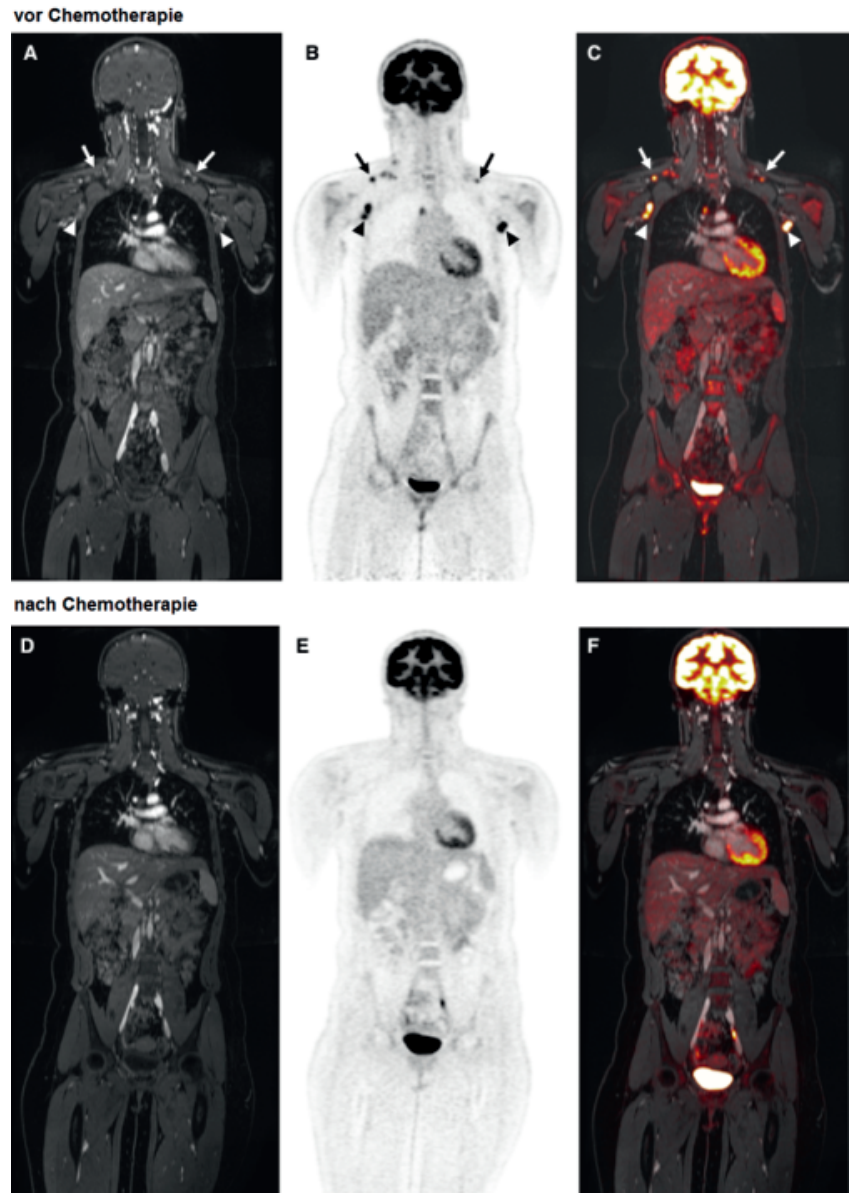

- Abb.5 14-jähriges Mädchen mit bioptisch gesichertem M. Hodgkin: A Die Kontrastmittel-unterstützte T1-gewichtete MRT zeigt vergrößerte mediastinale, supraklavikuläre und axilläre Lymphknoten (Pfeile). B Die simultan akquirierte FDG-PET zeigt einen erhöhten Tumormetabolismus. C Die simultane Datenakquisition ermöglicht eine exzellente Korrelation der FDG-PET und MRTDaten. D Die Kontrastmittel-unterstützte T1-gewichtete MRT, E simultan akquirierte FDG-PET und $\mathbf{F}$ integrierte PET/MRT zeigt eine Rückbildung der Lymphknoten nach Chemotherapie.

ADC-Werte ist jedoch komplex und noch nicht standardisiert oder validiert. Wenn diffusionsgewichtete Scans im Vergleich zur 18F-FDG-PET gleichwertige Informationen über die Therapiereaktion liefern können, kann dies neue Möglichkeiten eröffnen, andere, spezifischere PET-Tracer (z. B. 124I-MIBG) einzuführen.

\section{Fazit}

Zukünftige Entwicklungen werden sich darauf konzentrieren, die Strahlenbelastung weiter zu reduzieren, unnötige Untersuchungen zu vermeiden und den Ertrag der Bildgebung zu verbessern. Das enge Zusammenspiel von naturwissenschaftlichen Innovationen und klinischem Fortschritt werden eine wichtige Plattform für die Entwicklung neuartiger, moderner bildgebender Technologien bieten, um Diagnosen in der Pädiatrischen Radiologie sicherer, effizienter und akkurater zu gestalten. 

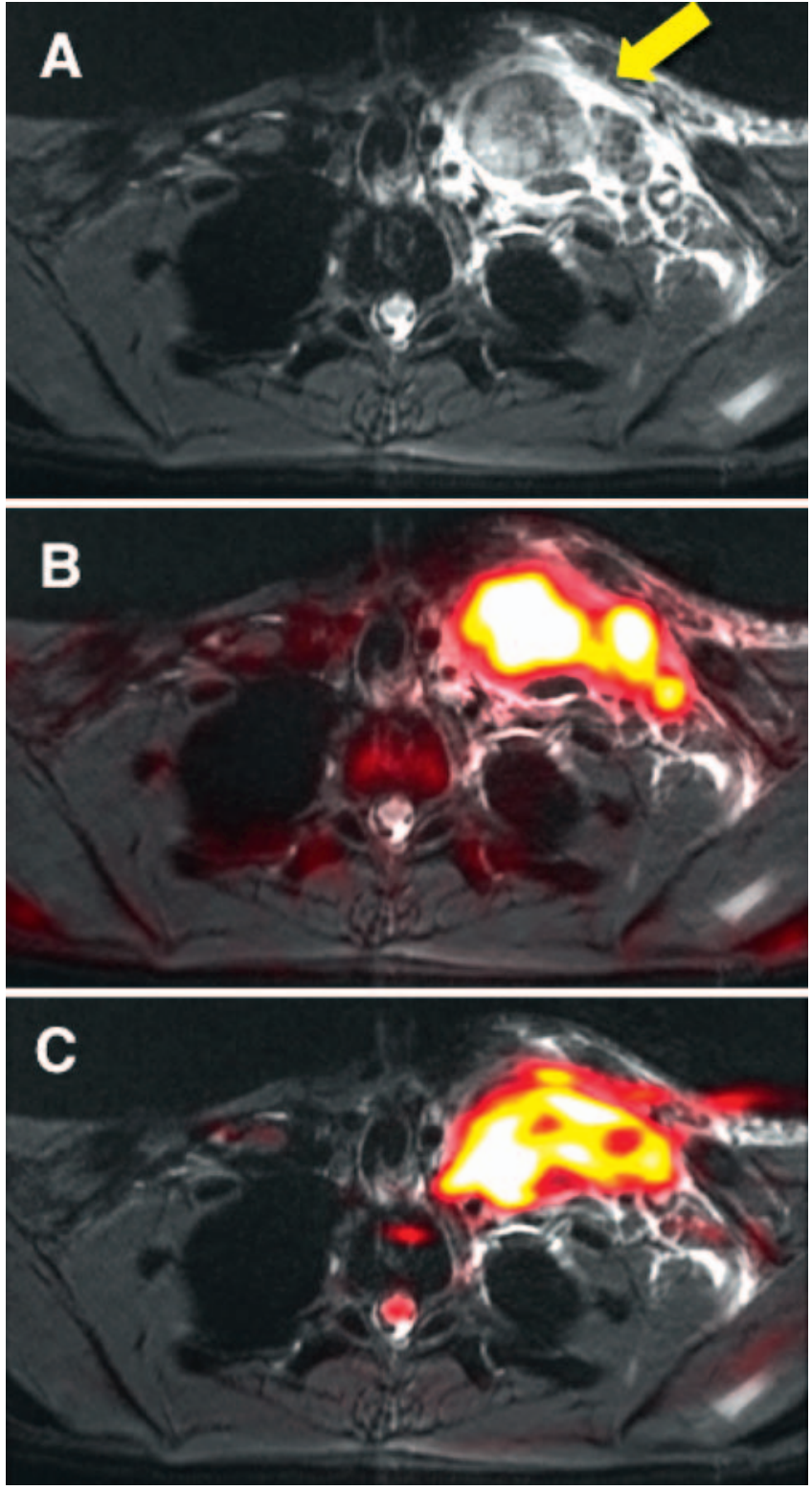

- Abb. 6 14-jähriger Junge mit bioptisch gesichertem M. Hodgkin: A Die Kontrastmittel-unterstützte T1-gewichtete MRT zeigt vergrößerte periklavikuläre Lymphknoten (Pfeil). B Die integrierte FDG-PET/MRT zeigt einen erhöhten Glukosemetabolismus in den vergrößerten Lymphknoten. C Die integrierte DWI/MRT ergibt einen ähnlichen Bildeindruck.

Interessenkonflikt

Die Autoren geben an, dass kein Interessenkonflikt besteht.

\section{Literatur}

[1] England JR, Cheng PM. Artificial Intelligence for Medical Image Analysis: A Guide for Authors and Reviewers. Am J Roentgenol 2018. doi:10.2214/ Am J Roentgenol.18.20490

[2] Lugo-Fagundo C, Vogelstein B, Yuille A et al. Deep Learning in Radiology: Now the Real Work Begins. J Am Coll Radiol 2018; 15: 364-367
[3] Wurfl T, Hoffmann M, Christlein V et al. Deep Learning Computed Tomography: Learning Projection-Domain Weights From Image Domain in Limited Angle Problems. IEEE Trans Med Imaging 2018; 37: 1454-1463

[4] Messerli M, Kluckert T, Knitel M et al. Ultralow dose CT for pulmonary nodule detection with chest $\mathrm{x}$-ray equivalent dose - a prospective intraindividual comparative study. Eur Radiol 2017; 27: 3290 - 3299

[5] Sorantin E, Weissensteiner S, Hasenburger $\mathrm{G}$ et al. CT in children-dose protection and general considerations when planning a CT in a child. Eur | Radiol 2013; 82: $1043-1049$

[6] Deak PD, Smal Y, Kalender WA. Multisection CT protocols: sex- and agespecific conversion factors used to determine effective dose from doselength product. Radiology 2010; 257: 158 - 166

[7] Sorantin E, Riccabona M, Stucklschweiger G et al. Experience with volumetric (320 rows) pediatric CT. Eur J Radiol 2013; 82: 1091 - 1097

[8] Daldrup-Link HE, Sammet C, Hernanz-Schulman M et al. White Paper on P4 Concepts for Pediatric Imaging. J Am Coll Radiol 2016; 13: 590 - 597. e592

[9] Ahmad R, Hu HH, Krishnamurthy R. Reducing sedation for pediatric body MRI using accelerated and abbreviated imaging protocols. Pediatr Radiol 2018; 48: 37-49

[10] Jaimes C, Kirsch JE, Gee MS. Fast, free-breathing and motion-minimized techniques for pediatric body magnetic resonance imaging. Pediatr Radiol 2018; 48: 1197 - 1208

[11] Feinberg DA, Setsompop K. Ultra-fast MRI of the human brain with simultaneous multi-slice imaging. J Magn Reson 2013; 229: 90 - 100

[12] Obele CC, Glielmi C, Ream J et al. Simultaneous Multislice Accelerated Free-Breathing Diffusion-Weighted Imaging of the Liver at 3T. Abdom imaging 2015; 40: 2323 - 2330

[13] Taron J, Martirosian P, Erb M et al. Simultaneous multislice diffusionweighted MRI of the liver: Analysis of different breathing schemes in comparison to standard sequences. J Magn Reson Imaging 2016; 44: $865-879$

[14] Lustig M, Donoho DL, Santos JM et al. Compressed Sensing MRI. IEEE Signal Processing Magazine 2008; 72: 72 - 82

[15] Lee JH, Choi YH, Cheon JE et al. Improved abdominal MRI in non-breathholding children using a radial k-space sampling technique. Pediatr Radiol 2015; 45: $840-846$

[16] Chandarana H, Block TK, Rosenkrantz AB et al. Free-breathing radial 3D fat-suppressed T1-weighted gradient echo sequence: a viable alternative for contrast-enhanced liver imaging in patients unable to suspend respiration. Invest Radiol 2011; 46: 648-653

[17] Goo HW, Ra YS. Advanced MRI for Pediatric Brain Tumors with Emphasis on Clinical Benefits. Korean J Radiol 2017; 18: 194-207

[18] Togao O, Yoshiura T, Keupp J et al. Amide proton transfer imaging of adult diffuse gliomas: correlation with histopathological grades. Neuro Oncol 2014; 16: 441 - 448

[19] Dillman JR, Trout AT, Merrow AC et al. Non-contrast three-dimensional gradient recalled echo Dixon-based magnetic resonance angiography/ venography in children. Pediatr Radiol 2018. doi:10.1007/s00247-0184297-3

[20] Kording F, Schoennagel BP, de Sousa MT et al. Evaluation of a Portable Doppler Ultrasound Gating Device for Fetal Cardiac MR Imaging: Initial Results at 1.5T and 3T. Magn Reson Med Sci 2018; 17: 308-317

[21] Schoennagel BP, Yamamura J, Kording F et al. Fetal dynamic phasecontrast MR angiography using ultrasound gating and comparison with Doppler ultrasound measurements. Eur Radiol 2019. doi:10.1007/ s00330-018-5940-y

[22] Strater A, Huber A, Rudolph J et al. 4D-Flow MRI: Technique and Applications. Fortschr Röntgenstr 2018; 190: 1025-1035

[23] Hernando D, Levin YS, Sirlin CB et al. Quantification of liver iron with MRI: state of the art and remaining challenges. J Magn Reson Imaging 2014; 40: 1003 - 1021 
[24] Reeder SB, Cruite I, Hamilton G et al. Quantitative assessment of liver fat with magnetic resonance imaging and spectroscopy. J Magn Reson Imaging 2011; 34: 729-749

[25] Dillman JR, Serai SD, Trout AT et al. Diagnostic performance of quantitative magnetic resonance imaging biomarkers for predicting portal hypertension in children and young adults with autoimmune liver disease. Pediatr Radiol 2019. doi:10.1007/s00247-018-4319-1

[26] Hamilton JI, Jiang $Y, C$ Chen $Y$ et al. MR fingerprinting for rapid quantification of myocardial T1, T2, and proton spin density. Magn Reson Med 2017; 77: 1446- 1458

[27] Chen Y, Chen MH, Baluyot KR et al. MR fingerprinting enables quantitative measures of brain tissue relaxation times and myelin water fraction in the first five years of life. Neuroimage 2019; 186: 782 - 793

[28] Ma D, Gulani V, Seiberlich N et al. Magnetic resonance fingerprinting. Nature 2013; 495: 187-192

[29] Kauczor HU, Kreitner KF. MRI of the pulmonary parenchyma. Eur Radiol 1999; 9: 1755-1764

[30] Tepper LA, Ciet P, Caudri D et al. Validating chest MRI to detect and monitor cystic fibrosis lung disease in a pediatric cohort. Pediatr Pulmonol 2016; 51: $34-41$

[31] Koumellis P, van Beek EJ, Woodhouse N et al. Quantitative analysis of regional airways obstruction using dynamic hyperpolarized $3 \mathrm{He}$ MRIpreliminary results in children with cystic fibrosis. J Magn Reson Imaging 2005; $22: 420-426$

[32] Walkup LL, Thomen RP, Akinyi TG et al. Feasibility, tolerability and safety of pediatric hyperpolarized (129)Xe magnetic resonance imaging in healthy volunteers and children with cystic fibrosis. Pediatr Radiol 2016; 46: 1651 - 1662

[33] Gutberlet M, Kaireit TF, Voskrebenzev A et al. Free-breathing Dynamic (19)F Gas MR Imaging for Mapping of Regional Lung Ventilation in Patients with COPD. Radiology 2018; 286: 1040 - 1051

[34] Hemberger KR, Jakob PM, Breuer FA. Multiparametric oxygen-enhanced functional lung imaging in 3D. Magma 2015; 28: 217-226

[35] Nikolaou K, Schoenberg SO, Brix G et al. Quantification of pulmonary blood flow and volume in healthy volunteers by dynamic contrast-enhanced magnetic resonance imaging using a parallel imaging technique. Invest Radiol 2004; 39: 537 - 545

[36] Eichinger M, Puderbach M, Fink C et al. Contrast-enhanced 3D MRI of lung perfusion in children with cystic fibrosis-initial results. Eur Radiol 2006; 16: $2147-2152$

[37] Stahl M, Wielputz MO, Graeber SY et al. Comparison of Lung Clearance Index and Magnetic Resonance Imaging for Assessment of Lung Disease in Children with Cystic Fibrosis. Am J Respir Crit Care Med 2017; 195: 349-359

[38] Martirosian P, Boss A, Fenchel M et al. Quantitative lung perfusion mapping at 0.2 T using FAIR True-FISP MRI. Magn Reson Med 2006; 55 : $1065-1074$

[39] Schraml C, Schwenzer NF, Martirosian P et al. Non-invasive pulmonary perfusion assessment in young patients with cystic fibrosis using an arterial spin labeling MR technique at 1.5 T. Magma 2012; 25: 155- 162

[40] Bauman G, Puderbach M, Deimling M et al. Non-contrast-enhanced perfusion and ventilation assessment of the human lung by means of fourier decomposition in proton MRI. Magn Reson Med 2009; 62: 656 664

[41] Kjorstad A, Corteville DM, Henzler T et al. Non-invasive quantitative pulmonary V/Q imaging using Fourier decomposition MRI at 1.5T. Z Med Phys 2015; 25: 326-332

[42] Higano NS, Fleck RJ, Spielberg DR et al. Quantification of neonatal lung parenchymal density via ultrashort echo time MRI with comparison to CT. J Magn Reson Imaging 2017; 46: 992 - 1000

[43] Sheikh K, Guo F, Capaldi DP et al. Ultrashort echo time MRI biomarkers of asthma. J Magn Reson Imaging 2017; 45: 1204-1215
[44] Fleischer S, Tsiflikas I, Langlouis V et al. Assessment of the Severity of Disease in Patients with Cystic Fibrosis using MRI of the Lung: Signal Intensity and Lung Volumes Compared to the Lung-Clearance-Index and Forced Expiratory-Volume-in-1-Second. Radiological Society of North America Scientific Assembly and Annual Meeting, Chicago IL 2016 November 27 - December 2, Chicago IL

[45] Daldrup-Link H, Gambhir SS. Pediatric Molecular Imaging. In: Treves ST, Hrsg Pediatric Nuclear Medicine and Molecular Imaging. Heidelberg: Springer; 2014: 671 - 596

[46] Willmann JK, Bonomo L, Carla Testa A et al. Ultrasound Molecular Imaging With BR55 in Patients With Breast and Ovarian Lesions: First-inHuman Results. J Clin Oncol 2017; 35: 2133-2140

[47] Perreault S, Ramaswamy V, Achrol AS et al. MRI surrogates for molecular subgroups of medulloblastoma. AJNR Am J Neuroradiol 2014; 35: $1263-1269$

[48] James ML, Gambhir SS. A molecular imaging primer: modalities, imaging agents, and applications. Phys Rev 2012; 92: 897-965

[49] Coleman JL, Navid F, Furman WL et al. Safety of ultrasound contrast agents in the pediatric oncologic population: a single-institution experience. Am J Roentgenol 2014; 202: 966 - 970

[50] Kiessling I, Bzyl ], Kiessling F. Molecular ultrasound imaging and its potential for paediatric radiology. Pediatr Radiol 2011; 41: 176-184

[51] Lindner JR. Molecular imaging with contrast ultrasound and targeted microbubbles. J Nucl Cardiol 2004; 11: 215-221

[52] Harmsen S, Teraphongphom N, Tweedle MF et al. Optical Surgical Navigation for Precision in Tumor Resections. Mol Imaging Biol 2017; 19 : $357-362$

[53] Shi C, Zhang C, Su Y et al. Cyanine dyes in optical imaging of tumours. Lancet Oncol 2010; 11: 815-816

[54] Lee H, Proudlock FA, Gottlob I. Pediatric Optical Coherence Tomography in Clinical Practice-Recent Progress. Invest Ophthalmol Vis Sci 2016; 57 : Oct69-79

[55] Uslu L, Donig J, Link $M$ et al. Value of 18F-FDG PET and PET/CT for evaluation of pediatric malignancies. J Nucl Med 2015; 56: 274-286

[56] Walter F, Czernin J, Hall T et al. Is there a need for dedicated bone imaging in addition to $18 \mathrm{~F}-\mathrm{FDG}$ PET/CT imaging in pediatric sarcoma patients? J Pediatr Hematol Oncol 2012; 34: 131 - 136

[57] Cheuk DK, Sabin ND, Hossain M et al. PET/CT for staging and follow-up of pediatric nasopharyngeal carcinoma. Eur J Nucl Med Mol Imaging 2012; 39: $1097-1106$

[58] Kleis M, Daldrup-Link H, Matthay K et al. Diagnostic value of PET/CT for the staging and restaging of pediatric tumors. Eur J Nucl Med Mol Imaging 2009; 36: $23-36$

[59] London K, Stege C, Cross S et al. 18F-FDG PET/CT compared to conventional imaging modalities in pediatric primary bone tumors. Pediatr Radiol 2012; 42: $418-430$

[60] Volker T, Denecke T, Steffen I et al. Positron emission tomography for staging of pediatric sarcoma patients: results of a prospective multicenter trial. J Clin Oncol 2007; 25: 5435- 5441

[61] Voss SD. Pediatric oncology and the future of oncological imaging. Pediatr Radiol 2011; 41 (Suppl. 1): S172 -S185

[62] McCarville MB. New frontiers in pediatric oncologic imaging. Cancer Imaging 2008; 8: 87-92

[63] Gallagher FA, Bohndiek SE, Kettunen MI et al. Hyperpolarized 13C MRI and PET: in vivo tumor biochemistry. J Nucl Med 2011; 52: 1333-1336

[64] Bohndiek SE, Kettunen MI, Hu DE et al. Hyperpolarized [1-13C]-ascorbic and dehydroascorbic acid: vitamin $\mathrm{C}$ as a probe for imaging redox status in vivo. J Am Chem Soc 2011; 133: 11795-11801

[65] Brindle KM, Bohndiek SE, Gallagher FA et al. Tumor imaging using hyperpolarized $13 \mathrm{C}$ magnetic resonance spectroscopy. Magn Reson Med 2011; 66: $505-519$ 
[66] Hu S, Balakrishnan A, Bok RA et al. 13C-pyruvate imaging reveals alterations in glycolysis that precede c-Myc-induced tumor formation and regression. Cell Metab 2011; 14: 131-142

[67] Daldrup-Link H. How PET/MR Can Add Value For Children With Cancer. Curr Radiol Rep 2017; 5: 15. Epub 2017

[68] Ponisio MR, McConathy J, Laforest R et al. Evaluation of diagnostic performance of whole-body simultaneous PET/MRI in pediatric lymphoma. Pediatr Radiol 2016; 46: 1258 - 1268

[69] Gawande RS, Gonzalez G, Messing S et al. Role of diffusion-weighted imaging in differentiating benign and malignant pediatric abdominal tumors. Pediatr Radiol 2013; 43: 836-845

[70] Klenk C, Gawande R, Uslu L et al. Ionising radiation-free whole-body MRI versus (18)F-fluorodeoxyglucose PET/CT scans for children and young adults with cancer: a prospective, non-randomised, single-centre study. Lancet Oncol 2014; 15: 275-285
[71] Bakhshi S, Radhakrishnan V, Sharma P et al. Pediatric nonlymphoblastic non-Hodgkin lymphoma: baseline, interim, and posttreatment PET/CT versus contrast-enhanced CT for evaluation-a prospective study. Radiology 2012; 262: $956-968$

[72] Riad R, Omar W, Kotb M et al. Role of PET/CT in malignant pediatric lymphoma. Eur J Nucl Med Mol Imaging 2010; 37: 319-329

[73] Bestic JM, Peterson J], Bancroft LW. Pediatric FDG PET/CT: Physiologic uptake, normal variants, and benign conditions [corrected]. Radiographics 2009; 29: $1487-1500$

[74] Stanescu L, Ishak GE, Khanna PC et al. FDG PET of the brain in pediatric patients: imaging spectrum with MR imaging correlation. Radiographics 2013; 33: $1279-1303$ 